\title{
Systemic and local injections of lupeol inhibit tumor growth in a melanoma-bearing mouse model
}

\author{
MAKIKO NITTA $^{1 *}$, KAZUO AZUMA $^{1 *}$, KEISHI HATA $^{2}$, SAORI TAKAHASHI $^{2}$, \\ KIKUMI OGIWARA ${ }^{3}$, TAKESHI TSUKA ${ }^{1}$, TOMOHIRO IMAGAWA ${ }^{1}$, INORU YOKOE ${ }^{1}$, \\ TOMOHIRO OSAKI ${ }^{1}$, SABURO MINAMI $^{1}$ and YOSHIHARU OKAMOTO ${ }^{1}$ \\ ${ }^{1}$ Faculty of Agriculture, Tottori University, Tottori, Tottori 6808553; ${ }^{2}$ Institute for Food and Brewing, \\ Akita Prefectural Agricultural, Forestry and Fisheries Research Center, Akita, Akita 0101623; \\ ${ }^{3}$ Laboratory of Veterinary Pathology, Azabu University, Sagamihara, Kanagawa 2525201, Japan
}

Received March 14, 2013; Accepted May 2, 2013

DOI: $10.3892 / b r .2013 .116$

\begin{abstract}
Melanoma is the most aggressive type of skin cancer and it is procured from activated or genetically altered epidermal melanocytes. In the present study, the tumor-suppressive effects of systemic and local injections of lupeol, a triterpene extracted from Indian lettuce (Lactuca indica), in a melanoma-bearing mouse model were evaluated. Mice were injected once with lupeol or olive oil (solvent control) subcutaneously into the skin of the back or into the tumor tissue. Seven days after the injection, the tumor growth rates were calculated and the tumor tissues were collected. Immunohistochemical staining for Ki-67 and proliferating cell nuclear antigen (PCNA) were performed. The tumor growth rates in the lupeol-injected group were significantly decreased compared to those observed in the non-treated (NT) and solvent control groups. Lupeol also significantly decreased the areas positively stained for Ki-67 and PCNA in the tumor tissues compared to those in the NT and solvent control groups. The results of the present study demonstrated that systemic and local injections of lupeol suppress tumor growth and induce cell cycle arrest in a melanoma-bearing mouse model. These data suggest that lupeol may be effective as a novel therapeutic option for melanoma patients.
\end{abstract}

\section{Introduction}

Melanoma is the most aggressive form of skin cancer and it is derived from activated or genetically altered epidermal

Correspondence to: Professor Yoshiharu Okamoto, Faculty of Agriculture, Tottori University, 4-101 Koyama Minami, Tottori, Tottori 6808553, Japan

E-mail: yokamoto@muses.tottori-u.ac.jp

*Contributed equally

Key words: lupeol, melanoma, systemic injection, anticancer, $\mathrm{Ki}-67$, proliferating cell nuclear antigen melanocytes (1). Human malignant melanoma is a highly metastatic cancer that is markedly resistant to chemotherapy with dacarbazine or temozolomide. The best single-agent activity achieves a response rate of $15-30 \%$ and a median duration of response of a few months (2). In the fields of veterinary medicine, canine melanoma is the most common oral malignant tumor (3).

Lupeol is a triterpene contained in olives, fruits such as mangos, strawberries, grapes and figs, numerous vegetables and several medicinal plants (4). Previous studies reported that lupeol possesses several bioactivities, such as anti-inflammatory (5), antioxidant (6) and antitumor effects $(7,8)$. In particular, lupeol has been described as being beneficial for inhibiting the proliferation of melanoma cells in vitro via several mechanisms of action, including differentiation- $(9,10)$ and apoptosis-inducing activities (11), activation of p38 mitogen-activated protein kinases (MAPK) (12), anti-angiogenic activities (13), remodeling of the actin cytoskeleton (14) and melanosome maturation (15).

A previous study by Saleem et al (11) reported that lupeol inhibits the growth of highly aggressive human metastatic melanoma cells in vivo by inducing apoptosis. They demonstrated the beneficial effects of lupeol in a melanoma mouse model using intraperitoneal injection of the compound. In that experiment, however, the injections of lupeol were initiated at the time of tumor cell transplantation. To the best of our knowledge, there is currently no report of the differences between lupeol injection routes and the effects of lupeol on tuberous tumor tissue in vivo. In order to evaluate the use of lupeol in the clinical treatment of melanoma patients, the effects of lupeol must be assessed using an easy administration route. In this study, we investigated the effects of lupeol via systemic and local administration in a tuberous melanoma-bearing mouse model.

\section{Materials and methods}

Reagent. In this study, we used lupeol extracted from Indian lettuce (Lactuca indica), [Hata et al (16)]. Olive oil was purchased from Wako Pure Chemical Industries, Ltd. (Osaka, 
Japan). Lupeol was dissolved in olive oil using heat $\left(37^{\circ} \mathrm{C}\right)$ and sonification $(3 \mathrm{~h})$. The concentration of dissolved lupeol was diluted to $5 \mathrm{mg} / \mathrm{ml}$.

Preparation of the melanoma-bearing mouse model. Forty female, 6-week-old C57BL/6 mice were purchased from CLEA Japan, Inc. (Osaka, Japan). The animals were maintained under conventional conditions. The use of these animals and the procedures they underwent were approved by the Animal Research Committee of Tottori University.

B16 2F2 melanoma cells were maintained in Dulbecco's modified Eagle's medium (DMEM) supplemented with $10 \%$ fetal bovine serum (FBS), $100 \mu \mathrm{g} / \mathrm{ml}$ streptomycin and $100 \mathrm{U} / \mathrm{ml}$ penicillin in an incubator at $37^{\circ} \mathrm{C}$ under a humidified atmosphere of $5 \% \mathrm{CO}_{2}$. The mice were anesthetized with inhalation of 3-5\% isoflurane (Intervet, Inc., Tokyo, Japan). A total of $1 \times 10^{6} \mathrm{~B} 162 \mathrm{~F} 2$ cells $\left(1 \times 10^{7}\right.$ cells $\left./ \mathrm{ml}\right)$ were subcutaneously injected into the dorsal regions of the mice. Mice whose tumors grew to $5 \mathrm{~mm}$ in size were used in this study.

Study design. The mice $(\mathrm{n}=40)$ were randomized into five groups: the non-treatment (NT) group, the subcutaneous injection of olive oil (solvent control) into the dorsal region $(\mathrm{C}$-si) group, the subcutaneous injection of lupeol into the dorsal region (L-si) group, the local injection of olive oil into the tumor tissue (C-li) group and the local injection of lupeol into the tumor tissue (L-li) group ( $n=8$ per group). Single injections of lupeol were systemically or locally administered to the mice (day 0 ). A total of $0.1 \mathrm{ml}$ of lupeol $(20 \mathrm{mg} / \mathrm{kg}$ ) was injected into each mouse. On day 7 after the injections the mice were euthanized with inhalation of 5\% isoflurane followed by cervical dislocation. The tumor growth rates were calculated according to the tumor volumes $\left(\mathrm{mm}^{3} /\right.$ day). On days 0 and 7 , the volumes of the tumor tissues were calculated by measuring the mediastinum, transverse lie and depth of each tumor. The tumors were removed and fixed in $10 \%$ buffered formalin.

$K i-67$ staining. Tissue sections $(3 \mu \mathrm{m})$ were placed on glass slides and were deparaffinized, washed with ethanol and water and soaked in phosphate-buffered saline (PBS). The sections were autoclaved with $0.01 \mathrm{M}$ citrate buffer ( $\mathrm{pH}$ 6.0) for $15 \mathrm{~min}$ at $121^{\circ} \mathrm{C}$, then washed with PBS and incubated with rabbit polyclonal anti-Ki-67 antibodies (1:50, code no. E0468, Dako, Glostrup, Denmark) for $30 \mathrm{~min}$ at room temperature. After being washed with PBS, the sections were incubated with rat anti-IgG antibodies (1:100, sc-372; Vector Laboratories, Inc., Burlingame, CA, USA) for $30 \mathrm{~min}$ at room temperature. The slides were washed with PBS and stained with the VECTASTAIN ABC kit (PK-4000; Vector Laboratories, Inc.) for $30 \mathrm{~min}$. The tissue sections were counterstained with HistoGreen (Nichirei Bioscience, Inc., Osaka, Japan) and then stained with nuclear fast red.

Proliferating cell nuclear antigen (PCNA) staining. Tissue sections $(3-\mu \mathrm{m})$ were placed on glass slides and were deparaffinized, washed with ethanol and water and soaked in PBS. The sections were treated using microwaves with distilled water for $5 \mathrm{~min}$, then washed with PBS and incubated with $1 \%$ hydrogen peroxide methanol for $30 \mathrm{~min}$ at room temperature. After being washed with PBS, the sections were incubated with Histofine MOUSESTAIN kit blocking reagent $\mathrm{A}$ (Nichirei Biosciences, Inc.) for $60 \mathrm{~min}$ at room temperature. The sections were then washed with PBS and incubated with rabbit polyclonal anti-PCNA antibodies (1:200, code no. M0879, Dako) for $60 \mathrm{~min}$ at room temperature. The slides were then incubated with Histofine MOUSESTAIN kit blocking reagent B (Nichirei Biosciences, Inc.) for $10 \mathrm{~min}$ at room temperature and were then washed with PBS and incubated with Histofine MOUSESTAIN kit-labeled polymer Max PO (Nichirei Biosciences, Inc.) for $10 \mathrm{~min}$ at room temperature. The tissue sections were counterstained with HistoGreen and then stained with nuclear fast red (Nichirei Biosciences, Inc.).

Image analysis of Ki-67 and PCNA staining. A quantitative digital morphometric analysis of the Ki-67- and PCNA-positive tumor areas was performed. In brief, 10 randomly selected high-power fields (x200 magnification) per cross section were photographed with a digital camera attached to an Olympus microscope system (Olympus Corporation, Tokyo, Japan). The color wavelengths of the copied images were transformed into digital readings using the Lumina Vision software program (Mitani Corporation, Tokyo, Japan), allowing for quantification of the various color wavelengths with pixels as the unit of measurement. The percentage of positive areas in the tumor tissues was calculated by dividing the total pixel area of the positive areas by the total pixel area corresponding to the total tumor tissue in the field of view. The tumor tissues of three mice were analyzed per group. The mean scores for 30 fields were used as the percentages of positive areas per group.

RNA extraction and complementary DNA (cDNA) synthesis. A total of $2.5 \times 10^{5} \mathrm{~B} 162 \mathrm{~F} 2$ melanoma cells were seeded and precultured in $1 \mathrm{ml}$ DMEM supplemented with $10 \%$ FBS in 12 -well plates for $12 \mathrm{~h}$. The cells were washed twice with PBS and incubated in the same medium for 2 days with or without $5 \mu \mathrm{M}$ of lupeol. Total RNA was isolated using the QuickGene RNA cultured cell kit S (Fujifilm Co., Tokyo, Japan). Template cDNA synthesis was performed with $5 \mu \mathrm{g}$ of total RNA using the PrimeScript RT reagent kit (Takara Bio Inc., Shiga, Japan).

Real-time reverse transcription polymerase chain reaction (qRT-PCR). In a Chromo4 fluorescent temperature cycler (Bio-Rad, Hercules, CA, USA), $2.5 \%$ of each RT reaction solution was amplified in $25 \mu \mathrm{l}$ of $1 \mathrm{X}$ SYBR Premix Ex Taq (Takara Bio Inc.) containing $0.2 \mu \mathrm{M}$ of each primer. The samples were incubated in the thermal cycler for an initial denaturation at $95^{\circ} \mathrm{C}$ for $10 \mathrm{sec}$ followed by 40 PCR cycles. Each cycle consisted of $95^{\circ} \mathrm{C}$ for $5 \mathrm{sec}$ and $60^{\circ} \mathrm{C}$ for $30 \mathrm{sec}$. The oligonucleotide primers used in the experiment are listed in Table I. To confirm the amplification of specific transcripts, melting curve profiles (cooling the sample to $60^{\circ} \mathrm{C}$ and reheating slowly to $95^{\circ} \mathrm{C}$ with continuous measurement of fluorescence) were produced at the end of each PCR cycle. The relative expression levels of the two mRNAs were normalized according to the amount of glyceraldehyde 3-phosphate dehydrogenase (GAPDH) mRNA (forward primer, 5'-TGTGTCCGTCGTGGATCTGA-3'; reverse primer, 5'-TTGCTGTTGAAGTCGCAGGAG-3'). 
Table I. Effects of lupeol on gene expression of B16 2F2 melanoma cells.

\begin{tabular}{|c|c|c|c|c|}
\hline \multirow[b]{2}{*}{ Gene } & \multicolumn{2}{|c|}{ Sequence } & \multicolumn{2}{|c|}{ RI/GAPDH (\%) } \\
\hline & Forward & Reverse & Control & Lupeol \\
\hline GUS & 5'-CTGTGACCGATACGGGATTGTG-3' & 5'-ACCTCTAGGTGGTGCCGAAGTG-3' & $100.0 \pm 2.8$ & $108.4 \pm 8.0$ \\
\hline Tyrosinase & 5'-CAAGTACAGGCATCGGCCAAC-3' & 5'-GGTGCATTGGCTTCTGGGTAA-3' & $100.0 \pm 6.4$ & $184.1 \pm 8.2^{\mathrm{a}}$ \\
\hline Rab27a & 5'-TAGCACTGCAGGGACGCAAC-3' & 5'-CAAGGCCAAGAACTTGATGAGGTAA-3' & $100.0 \pm 4.8$ & $123.9 \pm 14.5^{b}$ \\
\hline PCNA & 5'-GAGAGCTTGGCAATGGGAACA-3' & 5'-GGGCACATCTGCAGACATACTGA -3' & $100.0 \pm 5.3$ & $36.5 \pm 3.1^{\mathrm{a}}$ \\
\hline $\mathrm{Ki}-67$ & 5'-CGTGTCAAACAAACTTGAATCTGTG-3' & 5'-TCTGCAGATGCATCAAACTTGG-3' & $100.0 \pm 4.0$ & $39.9 \pm 4.0^{\mathrm{a}}$ \\
\hline
\end{tabular}

Data represent the mean \pm standard error of each group. Statistical significance was determined according to the Student's t-test; ${ }^{\text {aP }}<0.01$ and ${ }^{\mathrm{b}} \mathrm{P}<0.05$ compared to the control group. RI, relative intensity; GAPDH, glyceraldehyde 3-phosphate dehydrogenase; GUS, $\beta$-glucuronidase; PCNA, proliferating cell nuclear antigen.

Statistical analysis. Data were expressed as the mean \pm standard error or standard deviation. The statistical analyses were performed using the Steel-Dwass test or the Student's t-test. $\mathrm{P}<0.05$ was considered to indicate a statistically significant difference.

\section{Results}

Effects of lupeol via systemic and local administration on tumor growth and histopathological changes in the melanoma-bearing mouse model. The tumor growth rates are shown in Fig. 1. In the L-si group $\left(53.5 \pm 12.5 \mathrm{~mm}^{3} /\right.$ day $)$, the growth rates of the tumor tissues were significantly decreased compared to those observed in the NT $\left(177.5 \pm 36.4 \mathrm{~mm}^{3} /\right.$ day $)$ and C-si $\left(166.1 \pm 13.9 \mathrm{~mm}^{3} /\right.$ day $)$ groups $(\mathrm{P}<0.05$ vs. NT group and $\mathrm{P}<0.01$ vs. C-si group). In the L-li group $\left(41.8 \pm 12.7 \mathrm{~mm}^{3} /\right.$ day), the growth rates of the tumor tissues were significantly decreased compared to those observed in the NT group $(\mathrm{P}<0.05)$. In the L-li group, the growth rates of the tumor tissues were slightly decreased compared to those observed in the C-li group $\left(126.0 \pm 19.0 \mathrm{~mm}^{3} /\right.$ day $)$. There were no significant differences between the L-si and L-li groups.

Effects of lupeol via systemic and local administration on $\mathrm{Ki}$-67 staining in the melanoma-bearing mouse model. The results of the image analysis are shown in Fig. 2. In the L-si group $(10.2 \pm 1.0 \% /$ field $)$, the percentages of Ki-67-positive areas were significantly decreased compared to those observed in the NT $(21.1 \pm 1.4 \% /$ field $)$ and C-si $(22.4 \pm 0.8 \% /$ field $)$ groups $(\mathrm{P}<0.01)$. In the L-li group $(11.8 \pm 1.6 \% /$ field $)$, the percentages of Ki-67-positive areas were significantly decreased compared to those observed in the NT and C-li $(21.3 \pm 1.0 \% /$ field $)$ groups $(\mathrm{P}<0.01)$. There were no significant differences between the L-si and L-li groups.

Effects of lupeol via systemic and local administration on PCNA staining in the melanoma-bearing mouse model. The results of the image analysis are shown in Fig. 3. In the L-si group $(5.4 \pm 0.8 \% /$ field $)$, the percentages of PCNA-positive areas were significantly decreased compared to those observed in the NT (18.4 $\pm 1.1 \% /$ field) and C-si (15.2 $\pm 0.8 \% /$ field) groups $(\mathrm{P}<0.01)$. In the L-li group $(12.1 \pm 0.9 \% /$ field $)$, the percentages of PCNA-positive areas were significantly decreased compared

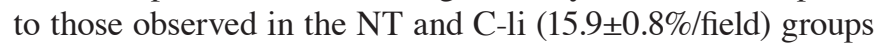

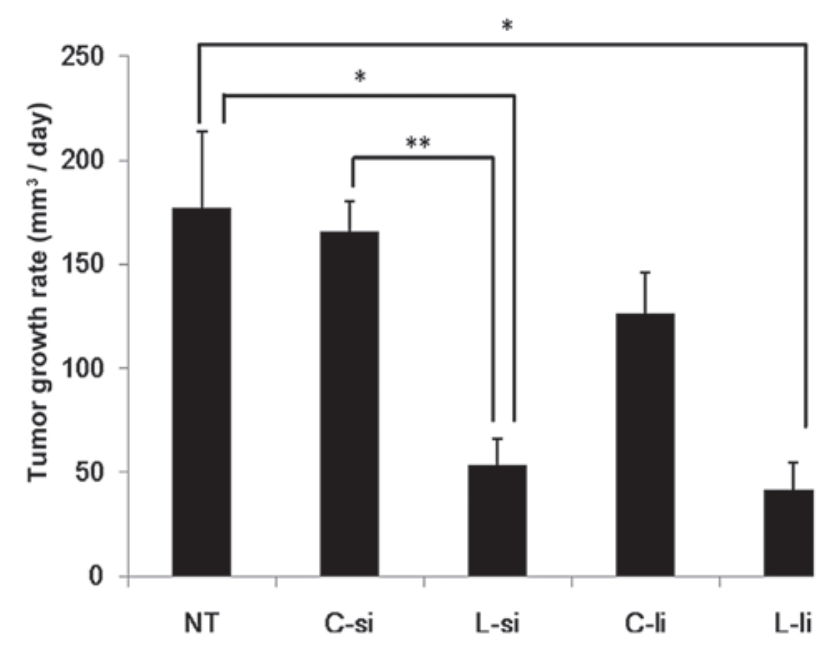

Figure 1. Effects of systemic and local administration of lupeol on tumor growth rate in the melanoma-bearing mouse model. The tumor volume was measured on days 0 and 7 . The tumor growth rates $\left(\mathrm{mm}^{3} /\right.$ day) were calculated according to the tumor volumes. Data represent the mean \pm standard error of each group. Statistical significance was determined according to the Steel-Dwass test; ${ }^{*} \mathrm{P}<0.05$ and ${ }^{* *} \mathrm{P}<0.01$. NT, non-treatment group; C-si, solvent control group; L-si, subcutaneous injection of lupeol into the dorsal region group; C-li, local injection of olive oil into the tumor tissue group; L-li; local injection of lupeol into the tumor tissue group.

( $\mathrm{P}<0.01$ vs. NT group and $\mathrm{P}<0.05$ vs. $\mathrm{C}$-li group). There were no significant differences between the L-si and L-li groups.

Effects of lupeol on the expression of markers of melanoma cell differentiation and proliferation. Administration of $5 \mu \mathrm{M}$ of lupeol did not affect the gene expression of $\beta$-glucuronidase (GUS, a housekeeping gene) in the B16 2F2 cells. The markers of pigment cell differentiation tyrosinase and Rab27a were elevated by $5 \mu \mathrm{M}$ of lupeol at the mRNA level; however, the agent markedly attenuated the expression of the PCNA and Ki-67 genes (Table I).

\section{Discussion}

Several previous studies indicated that lupeol exhibits antitumor activities against melanoma cells in vitro $(9,11-16)$. A previous study by Saleem et al (11) demonstrated that intraperitoneal injection of lupeol inhibits tumor growth in a human 


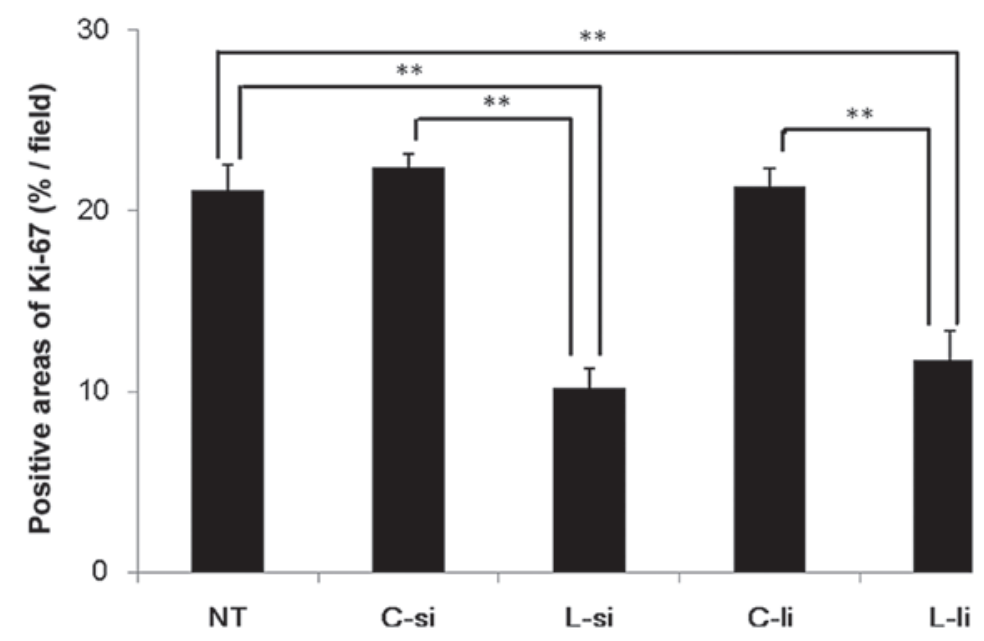

Figure 2. Effects of systemic and local administration of lupeol on immunohistochemical staining of Ki-67. Tumor tissues were fixed and immunohistochemical staining for Ki-67 was performed. The percentages of the Ki-67-positive areas are represented by the bars. Data represent the mean \pm standard error of each group. Statistical significance was determined according to the Steel-Dwass test; ${ }^{* *} \mathrm{P}<0.01$. NT, non-treatment group; C-si, solvent control group; L-si, subcutaneous injection of lupeol into the dorsal region group; C-li, local injection of olive oil into the tumor tissue group; L-li; local injection of lupeol into the tumor tissue group.

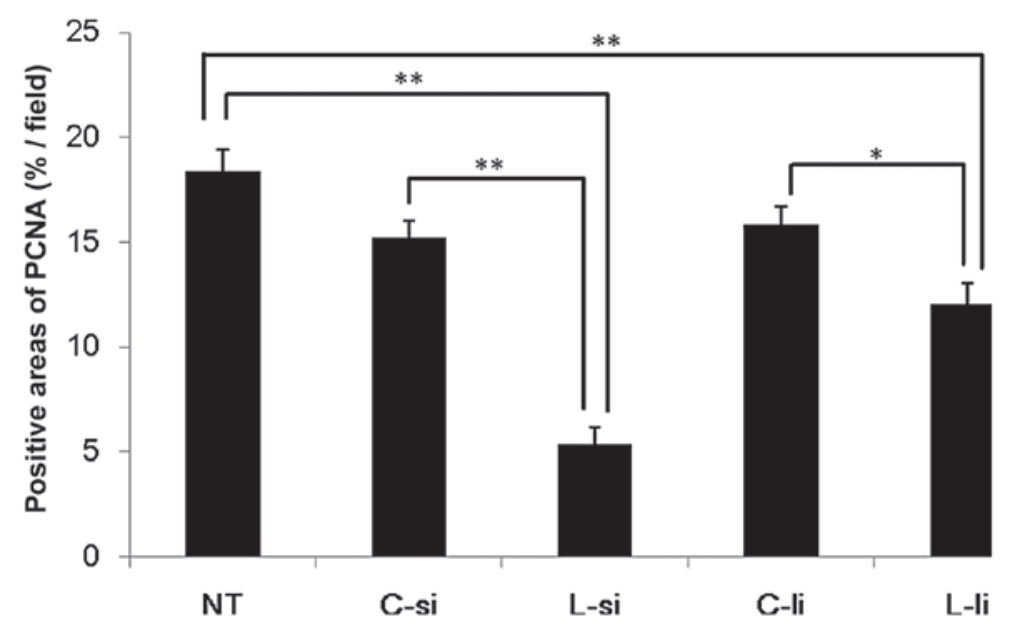

Figure 3. Effects of systemic and local administration of lupeol on immunohistochemical staining of proliferating cell nuclear antigen (PCNA). Tumor tissues were fixed and immunohistochemical staining for PCNA was performed. The percentages of the PCNA-positive areas are represented by the bars. Data represent the mean \pm standard error of each group. Statistical significance was determined according to the Steel-Dwass test; ${ }^{*} \mathrm{P}<0.05$ and ${ }^{* * *} \mathrm{P}<0.01$. NT, non-treatment group; C-si, solvent control group; L-si, subcutaneous injection of lupeol into the dorsal region group; C-li, local injection of olive oil into the tumor tissue group; L-li; local injection of lupeol into the tumor tissue group.

melanoma-bearing mouse model. To incorporate lupeol into the clinical treatment of melanoma patients, its effects must be evaluated using an easy administration route. In the present study it was demonstrated that systemic and local administration of lupeol inhibits tumor growth in a melanoma-bearing mouse model.

In this study, systemic and local administration of lupeol decreased the percentage of Ki-67- and PCNA-positive areas in the tumor tissues. Ki-67 is a cell proliferation marker that is detected during all the active phases of the cell cycle and is absent in resting cells (17). The levels of Ki-67 increase during the synthesis phase (S phase) until mitosis, when its expression reaches a maximum. After cell division, cells in the G1 phase exhibit a decrease in the Ki-67 expression until they re-enter the $\mathrm{S}$ phase, when the levels of $\mathrm{Ki}-67$ increase again (18). PCNA is also a cell proliferation marker whose levels increase progressively from the G1 to the S phase (19). In numerous melanoma patients the expression of $\mathrm{Ki}-67$ and PCNA in the tumor tissue is correlated with the presence of malignancy and prognosis (20). The intraperitoneal injection of lupeol has been reported to reduce the expression of Ki-67 and PCNA in tumor tissue (11). Our data indicated similar results, suggesting that systemic and local administration of lupeol is associated with efficient cell cycle arrest of melanoma cells.

The effects of lupeol on the expression of markers of melanoma cell differentiation and proliferation were investigated (Table I). The markers of pigment cell differentiation, tyrosinase and Rab27a (15) were elevated by lupeol at the mRNA level; however, the agent markedly attenuated the expression of the PCNA and Ki-67 genes. These results suggest that lupeol suppresses PCNA and Ki-67 by inducing B16 2F2 
cell differentiation and they are in agreement with the results shown in Figs. 2 and 3.

Several treatment methods have been used for melanoma patients, including surgical resection, chemotherapy and radiation therapy (21). Recently, high-dose interferon- $\alpha$ therapy, high-dose interleukin-2 therapy, antibody blockade of cytotoxic T-lymphocyte-associated antigen 4 , inhibitors of the MAPK pathway and adoptive cell therapy have also been used in the treatment of melanoma (20-22). Our results indicate that the systemic and local administration of lupeol may be an effective novel therapeutic option for melanoma patients.

\section{Acknowledgements}

This study was supported by a grant from the Ministry of Education, Sports, Culture, Science and Technology (no. 20580352).

\section{References}

1. Oliveria S, Dusza S and Berwick M: Issues in the epidemiology of melanoma. Expert Rev Anticancer Ther 1: 453-459, 2001.

2. Korn EL, Liu PY, Lee SJ, Chapman JA, Niedzwiecki D, Suman VJ, Moon J, Sondak VK, Atkins MB, Eisenhauer EA, et al: Meta-analysis of phase II cooperative group trials in metastatic stage IV melanoma to determine progression-free and overall survival benchmarks for future phase II trials. J Clin Oncol 26: 527-534, 2008

3. Bergman PJ: Canine oral melanoma. Clin Tech Small Anim Pract 22: 55-60, 2007.

4. Saleem M, Kaur S, Kweon MH, Adhami VM, Afaq F and Mukhtar H: Lupeol, a fruit and vegetable based triterpene, induces apoptotic death of human pancreatic adenocarcinoma cells via inhibition of Ras signaling pathway. Carcinogenesis 26: 1956-1964, 2005.

5. Geetha T and Varalakshmi P: Anti-inflammatory activity of lupeol and lupeol linoleate in rats. J Ethnopharmacol 76: 77-80, 2001.

6. Prasad S, Kalra N and Shukla Y: Hepatoprotective effects of lupeol and mango pulp extract of carcinogen induced alteration in Swiss albino mice. Mol Nutr Food Res 51: 352-359, 2007.
7. Chaturvedi PK, Bhui K and Shukla Y: Lupeol: connotations for chemoprevention. Cancer Lett 263: 1-13, 2008.

8. Saleem M: Lupeol, a novel anti-inflammatory and anti-cancer dietary triterpene. Cancer Lett 285: 109-115, 2009.

9. Hata K, Ishikawa K, Hori K and Konishi T: Differentiationinducing activity of lupeol, a lupane-type triterpene from Chinese dandelion root (Hokouei-kon), on a mouse melanoma cell line. Biol Pharm Bull 23: 962-967, 2000.

10. Hata K, Mukaiyama T, Tsujimura N, Sato Y, Kosaka Y, Sakamoto $\mathrm{K}$ and Hori K: Differentiation-inducing activity of lupane triterpenes on a mouse melanoma cell line. Cytotechnology 52: 151-158, 2006.

11. Saleem M, Maddodi N, Abu Zaid M, Khan N, bin Hafeez B, Asim M, Suh Y, Yun JM, Setaluri V and Mukhtar H: Lupeol inhibits growth of highly aggressive human metastatic melanoma cells in vitro and in vivo by inducing apoptosis. Clin Cancer Res 14: 2119-2127, 2008.

12. Hata K, Hori K and Takahashi S: Role of p38 MAPK in lupeol-induced B16 2F2 mouse melanoma cell differentiation. J Biochem 134: 441-445, 2003.

13. You YJ, Nam NH, Kim Y, Bae KH and Ahn BZ: Antiangiogenic activity of lupeol from Bombax ceiba. Phytother Res 17: 341-344, 2003.

14. Hata K, Hori K, Murata J and Takahashi S: Remodeling of actin cytoskeleton in lupeol-induced B16 2F2 cell differentiation. J Biochem 138: 467-472, 2005.

15. Ogiwara K and Hata K: Melanoma cell differentiation induced by lupeol separates into two stages: morphological and functional changes. J Nat Med 63: 323-326, 2009.

16. Hata K, Hori K, Mukaiyama T, Sakamoto K and Takahashi S: Activators of melanin biosynthesis from Lactuca indica. Nat Med 57: 238-241, 2003.

17. Brown DC and Gatter KC: Ki67 protein: the immaculate deception? Histopathology 40: 2-11, 2002.

18. Lopez F, Belloc F, Lacombe F, Dumain P, Reiffers J, Bernard P and Boisseau MR: Modalities of synthesis of Ki67 antigen during the stimulation of lymphocytes. Cytometry 12: 42-49, 1991.

19. Moldovan GL, Pfander B and Jentsch S: PCNA, the maestro of the replication fork. Cell 129: 665-679, 2007.

20. Gould Rothberg BE, Bracken MB and Rimm DL: Tissue biomarkers for prognosis in cutaneous melanoma: a systematic review and meta-analysis. J Natl Cancer Inst 101: 452-474, 2009.

21. Lee B, Mukhi N and Liu D: Current management and novel agents for malignant melanoma. J Hematol Oncol 5: 3, 2012.

22. Garbe C, Eigentler TK, Keilholz U, Hauschild A and Kirkwood JM: Systematic review of medical treatment in melanoma: current status and future prospects. Oncologist 16: $5-24,2011$. 